\title{
Experimental detection of gravitational waves
}

\section{Lukanenkov A.V.}

Moscow, Russian Federation;

E-mail: Lukanenkov<a_v_luk@mail.ru>;

Relative displacements $\left(\approx 10^{-15} \mathrm{~m}\right)$ of different points on the Earth are detected using the optimal data of global seismic antenna, corresponding signals lie in planes perpendicular to the direction of the radiation source, have a high degree of elliptical polarization.

The fact of registration gravitational wave confirmed by the detection of gravitational signals for a long time (90 hours) in a frequency band near $6.023 \mathrm{~Hz}$.

Confidence probability of detection of gravitational wave is close to 1, it causes deformation (curvature) of the space order of $10^{-21}$.

The pulsar J0945-4833 is the most probable source of detected GW-signals, on gravitational radiation expended about $\varepsilon \approx 10^{-5}$ on the total energy of the pulsar.

Keywords: gravitational waves, gravitational signal, gravitational detector, seismic antenna, seismic station, deformation, elliptical polarization.

DOI: $10.18698 / 2309-7604-2015-1-343-358$

\section{Introduction}

Gravitational waves $(\mathrm{GW})$ are an inevitable consequence of the many theories of gravity [1,2]. Indirectly, GW were identified in the motion of binary pulsars [3].

Gravitational wave stretches and compresses space [1,2]. It causes a relative oscillatory motion of different points of space and it can be registered.

Many works devoted to experiments on the detection of gravitational waves, including projects based on laser interferometers (LIGO, VIRGO et al.) [1-8].

As a gravity detector is proposed to use the global seismic antenna (GSA), its elements arbitrary "quiet" seismic stations, the aperture should be about 10,000 km. Registration the relative movement of different points on the Earth's surface implemented as a result of optimal processing data of global seismic antenna (GSA) $[9,10]$. Using the Earth as a detector of gravitational waves is explained in Appendix A.

In this paper we consider the variant of GSA, based on 19 seismic stations of the International Monitoring System (IMS) of the Comprehensive Nuclear Test Ban Treaty (CTBT), placed on different continents (Figure 1) [11]. 


\section{The mathematical model of the gravitational signal}

If gravitational wave falls on seismometer (detector) with an angular frequency $\omega=2 \pi f$ in the direction $z$, then in its own local frame motion seismometer mass element under the action of gravitational waves is described by equations (TT coordinates) [2,12]:

$$
\left\{\begin{array}{l}
\frac{d^{2} x}{d t^{2}}=\frac{1}{2}\left(\ddot{A}_{+}\left(t-\frac{z}{c}\right) x+\ddot{A}_{\times}\left(t-\frac{z}{c}\right) y\right) \\
\frac{d^{2} y}{d t^{2}}=\frac{1}{2}\left(-\ddot{A}_{+}\left(t-\frac{z}{c}\right) y+\ddot{A}_{\times}\left(t-\frac{z}{c}\right) x\right) \\
\frac{d^{2} z}{d t^{2}}=0, \\
h_{x 0 x 0}^{T T}=-h_{y 0 y 0}^{T T}=A_{+}\left(t-\frac{z}{c}\right), h_{x 0 y 0}^{T T}=h_{y 0 x 0}^{T T}=A_{\times}\left(t-\frac{z}{c}\right) \text { - perturbation metric. }
\end{array}\right.
$$

If the source of the GW-periodic waves, the perturbations of the metric also are periodic functions. Therefore, solving the system of equations (1) are also periodic functions:

$$
x(t)=x(t+T), y(t)=y(t+T), T=1 / f
$$

and they can be expanded in a Fourier series:

$$
\begin{aligned}
& x(t)=\sum_{n=1}^{\infty} a_{x}(\mathrm{n}) \cos (\mathrm{n} \cdot 2 \pi f t)+b_{x}(\mathrm{n}) \cdot \sin (\mathrm{n} \cdot 2 \pi f t) \\
& y(t)=\sum_{n=1}^{\infty} a_{y}(\mathrm{n}) \cos (\mathrm{n} \cdot 2 \pi f t)+b_{y}(n) \cdot \sin (\mathrm{n} \cdot 2 \pi f t)
\end{aligned}
$$

First approximation of these solutions are harmonic components $(n=1)$ : 


$$
\begin{aligned}
& x_{1}(t)=a_{x}(1) \cos (2 \pi f t)+b_{x}(1) \cdot \sin (2 \pi f t) \\
& y_{1}(t)=a_{y}(1) \cos (2 \pi f t)+b_{y}(1) \cdot \sin (2 \pi f t)
\end{aligned}
$$

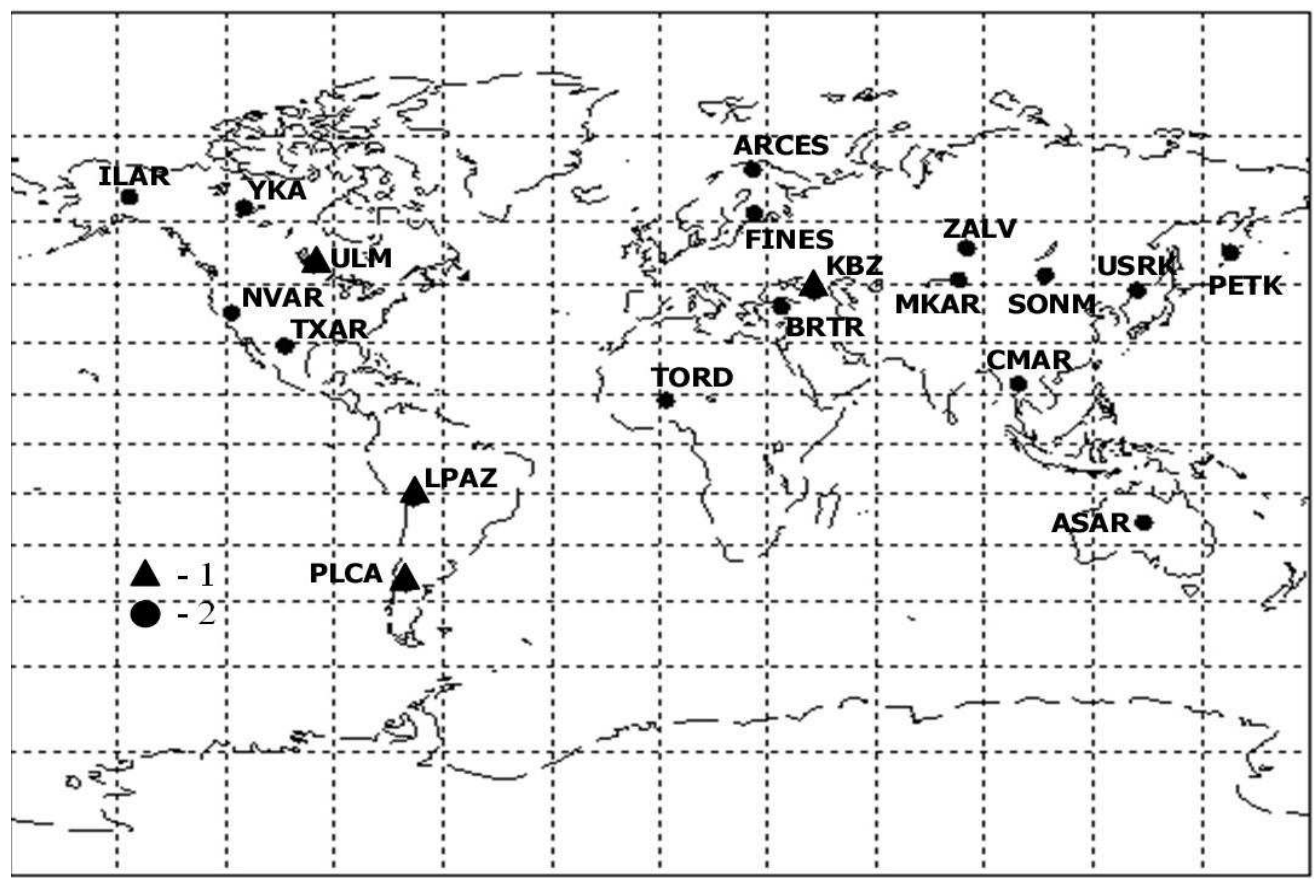

Fig. 1. Location of selected IMS stations CTBT [5].

1 - three-component station; 2 - seismic array.

For an arbitrary direction of propagation of gravitational-wave signals registered are represented as:

$$
\begin{gathered}
\mathbf{s}(\mathbf{r}, t)=\mathrm{a}_{\text {disp }}(f, \mathbf{p}) \cos (2 \pi \mathrm{f}(\mathrm{t}+\tau)) \mathbf{e}_{1}+b_{\text {disp }}(f, \mathbf{p}) \sin (2 \pi f(t+\tau)) \mathbf{e}_{2}, \\
\tau=(\mathbf{k}, \mathbf{p}), k=\mathbf{r} / c, \\
\mathrm{~S}(\mathbf{r}, \mathrm{t})=\left(S_{1}(\mathbf{r}, t), S_{2}(\mathbf{r}, t), 0\right), \mathbf{S}(\mathbf{r}, t) \in \mathrm{L}\left(\mathbf{e}_{1}, \mathbf{e}_{2}\right), \mathbf{e}_{1} \perp \mathbf{p}, \mathbf{e}_{2} \perp \mathbf{p}, \\
\mathrm{S}_{1}(\mathbf{r}, t)=\mathrm{a}_{\text {disp }}(\mathrm{f}, \mathbf{p}) \cos (2 \pi f(t+\tau)), S_{2}(\mathbf{r}, t)=\mathrm{b}_{\text {disp }}(f, \mathbf{p}) \sin (2 \pi f(t+\tau)),
\end{gathered}
$$

orthonormal basis $\left(\mathbf{e}_{1}, \mathbf{e}_{2}, \mathbf{p}\right)$; 
$\mathbf{r}=\mathbf{r}(\varphi, \lambda)=R_{3}(\cos \varphi \cos \lambda, \cos \varphi \sin \lambda, \sin \varphi)-$ vector from the center of the Earth to the point on the surface with coordinates $(\varphi, \lambda)$; Earth radius $R_{E}=6371000 \mathrm{~m}$;

$$
\mathbf{p}=\left(\cos \varphi_{\mathbf{p}} \cos \lambda_{\mathbf{p}}, \cos \varphi_{\mathbf{p}} \sin \lambda_{\mathbf{p}}, \sin \varphi_{\mathbf{p}}\right)-\text { vector directed to the point of }
$$

the celestial sphere $\left(\varphi_{p}, \lambda_{p}\right)$ (second equatorial coordinate system).

Signal (2) - the ellipse in the plane perpendicular to the direction $\mathbf{p}$, semiaxis $a_{\text {disp }}(f, \mathbf{p})$ и $b_{\text {disp }}(f, \mathbf{p})$.

\section{Detector gravitational signals}

For the detection of weak GW - signals appropriate to apply the methods of optimal data processing seismic network. The detector must perform the detection of elliptically polarized signal to background seismic noise.

Data recorded by GSA represented as the vector of observations:

$$
\vec{y}(m)=\left(\mathbf{y}_{1}(m), \ldots \mathbf{y}_{i}(m), \ldots, \mathbf{y}_{N_{s t}}(m)\right)^{T}
$$

Where $\mathbf{y}_{i}(m)=\mathbf{s}\left(\mathbf{r}_{i}, m \Delta t\right)+\mathbf{n}\left(\mathbf{r}_{i}, m \Delta t\right), 1 \leq m \leq N$;

$\mathbf{y}_{i}(m)$ - registrable seismic wave field (SWF) on the $\boldsymbol{i}$-th station;

$\mathbf{s}\left(\mathbf{r}_{i}, t\right)$-detected harmonic signal on the $\boldsymbol{i}$-th station;

$\mathrm{n}\left(\mathbf{r}_{i}, \mathrm{t}\right)$ - the seismic noise on the $\boldsymbol{i}$-th station;

$\mathbf{r}_{i}$-vector from the center of the earth to the point of placing of the $\boldsymbol{i}$-th station;

$\boldsymbol{N}_{\text {st }}$ - number of stations seismic network; $\boldsymbol{N}$ - size of the sample data;

$\Delta t$ - sampling interval time, $\boldsymbol{F}_{\mathrm{d}}=1 / \Delta t$ - sampling frequency.

Characteristics and properties of seismic noise [13 -17]:

- Normal distribution of seismic noise;

- Noise on seismic stations are independent, if stations are spaced apart from one another at distances of several hundred kilometers or more;

- Power spectral density $G_{n}(f) \leq 10^{-18}(\mathrm{~m} / \mathrm{s})^{2} / \mathrm{Hz}$ to "quiet" seismic arrays on total beam;

- Power spectral density decreases with increasing frequency $G_{n}(f) \approx G_{n}(1) \cdot f^{-2}$;

- Stationary seismic noise interval $\approx 6-8$ hours in the frequency band less than $0.1 \mathrm{~Hz}$. 
Functional optimal method of detecting the signal of the form (2) can be represented in the form of log-likelihood ratio:

$$
L(\vec{y} / s)=\ln \frac{P(\vec{y} / s)}{P(\vec{y} / n)},
$$

where $\vec{y}=\left(\mathbf{y}_{1}(t), \ldots, \mathbf{y}_{i}(t), \ldots, \mathbf{y}_{N_{s t}}(t)\right)$;

$\mathbf{y}_{i}(t)$ - registrable SWF on the $i$-th station;

$P(\vec{y} / s)(\mathrm{P}(\vec{y} / n))$ - joint distribution density $\vec{y}$ provided that availability detectable signal, or lack thereof, respectively.

Since the seismic noise are independent for different stations, the functional (3) can be written as:

$$
L(\vec{y} / s)=\ln \prod_{i=1}^{N_{s t}} \frac{P\left(\vec{y}_{i} / s\right)}{P\left(\vec{y}_{i} / n\right)}=\sum_{i=1}^{N_{s t}} \ln \frac{P\left(\vec{y}_{i} / s\right)}{P\left(\vec{y}_{i} / n\right)}
$$

where $P\left(\vec{y}_{i} / s\right)\left(P\left(\vec{y}_{i} / n\right)\right)$ - the density $y_{i}(t)$, subject to the signal or lack thereof.

Optimal processing functional when it detects a priori unknown signal of the form (2) is [9]

$$
L_{m}(\vec{y})=\max _{\theta} L(\vec{y} / \theta),
$$

where $\theta=\{a(f, \mathbf{p}), b(f, \mathbf{p}), \mathbf{P}\}-$ set of signal parameters of the form (2)

Optimal functional estimates the energy of gravitational radiation coming from an arbitrary point on the celestial sphere $\left(\varphi_{\mathbf{p}}, \lambda_{\mathbf{p}}\right)$ at frequency $\boldsymbol{f}$ :

$$
\begin{gathered}
E(f, \mathbf{p})=a_{V}(f, \mathbf{p})^{2}+b_{V}(f, \mathbf{p})^{2} \\
a_{V}(f, \mathbf{p})=\omega \cdot \mathrm{a}_{\text {disp }}(f, \mathbf{p}), b_{V}(f, \mathbf{p})=\omega \cdot b_{\text {disp }}(f, \mathbf{p}),
\end{gathered}
$$

i.e. the energy estimation of elliptically polarized seismic process (EPSP),

$\left(a_{v}(f, \mathbf{p})\right.$ и $\left.b_{v}(f, \mathbf{p})\right)$ - semiaxes of the ellipse along speed. 
Estimation of energy is carried out on the time fragment $\left[t_{0}, t_{0}+T\right]$, whereby $E(f, \mathbf{p})$ is a function of the duration time $t_{0}$ and $T$. To estimate the energy 32 directions (beams) $\mathbf{p}$ were chosen:

$$
\begin{aligned}
& \phi_{\mathrm{p}}=-75^{\circ}+\left(k_{\phi}-1\right) d \phi, \lambda_{\mathrm{p}}=\left(k_{\lambda}-1\right) d \lambda, k_{\phi}=1 \div 4, k_{\lambda}=1 \div 8, \mathrm{~d} \phi=45^{\circ}, \\
& d \lambda=22.5^{\circ}
\end{aligned}
$$

Earlier, using the optimal processing seismic data of the global seismic antenna $(T=8$ hours), seven signals were detected with the confidence probability $P_{\text {conf }}=1-\alpha>0.97$ [9]. These signals describe the relative motion of the points of seismic stations placement on different continents of the Earth.

These signals lie in planes perpendicular to the direction on certain points of the celestial sphere, have elliptical polarization, the degree of polarization of $>90 \%$ [9]. These signals corresponding wave describes the relative motion $\left(\approx 10^{-15} \mathrm{~m}\right)$ different points of the Earth distances of several thousand kilometers (4,000 to $11,000 \mathrm{~km})$, the theoretical justification of the possibility of registering such levels found in $[9,10]$.

They cause deformation (strain) of $h \approx 10^{-21}$, wave propagation velocity $v_{\mathrm{S}}>1.7 \cdot 10^{8} \mathrm{~m} / \mathrm{s}$ [9].

Next, we consider estimates of $E(f, \mathbf{p})$ on the 4-hour fragments ( $T=4$ hours).

Averaging the values of the energies at a frequency $f$ in space, we estimate the energy received by the antenna at a given frequency $E_{\text {aver }}\left(f, t_{0}\right)=\frac{1}{32} \sum_{\mathbf{p}} E(f, \mathbf{p})$ on the fragment $\left[t_{0}, t_{0}+T\right]$.

Estimating the energy on time fragments ( shift $=1$ hour), you can get a diagram of spectral-time analysis , $0 \leq t_{0} \leq 90$ hours ,frequency step $d f=40 / 4096 \mathrm{~Hz}$ (Fig. 2). They vary in the range from $1 \cdot 10^{-27}(\mathrm{~m} / \mathrm{s})^{2}$ to $35 \cdot 10^{-27}(\mathrm{~m} / \mathrm{s})^{2}$. 


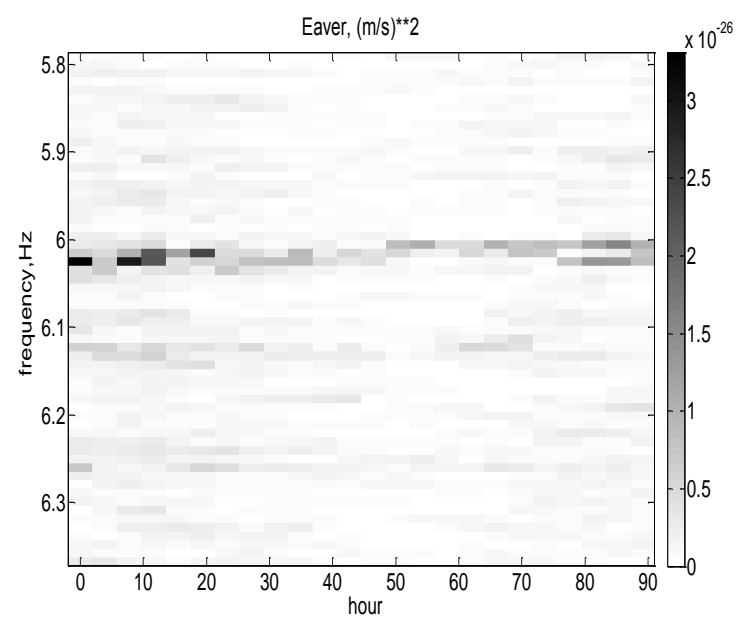

Fig. 2.The average energies EPSP $E_{\text {aver }}\left(f, t_{0}\right)$ for $00^{00} 27.02 .2009-18^{00} 2.03 .2009$.

Bandwidth $[5.8 \div 6.4 \mathrm{~Hz}]$.

It is also possible to estimate the average spectrum of energy for 90 hours observation:

$$
E_{\text {av }}(f)=\frac{1}{90} \sum_{t_{0}=0}^{89} E_{\text {aver }}\left(f, t_{0}\right),
$$

$E_{\text {av }}(f)$ - the average energies EPSP in time and space.

Analyzing the Figure 2, one can observe a well-defined signal within 90 hours ( $\approx 4$ days) in the band from 5.99 to $6.05 \mathrm{~Hz}$ with a center frequency $f_{0}=6.023 \mathrm{~Hz}$.

\section{Confidence probability of detection}

Assessing the significance level (the error of the $1^{\text {st }}$ kind), it is possible to confirm the hypothesis that the fact that the ejection of energy at the frequency $f_{0}=6.023 \mathrm{~Hz}$ is not random.

Spectrum $E_{\text {av }}(f)$ (Fig. 3) is the result of averaging 90 energy spectra (including strictly $90 / 4 \approx 22$ independent spectra and equal to the number of spectra on disjoint time intervals), and therefore we can assume that $E_{\mathrm{av}}(f)$ is distributed in almost normal law at each frequency. 


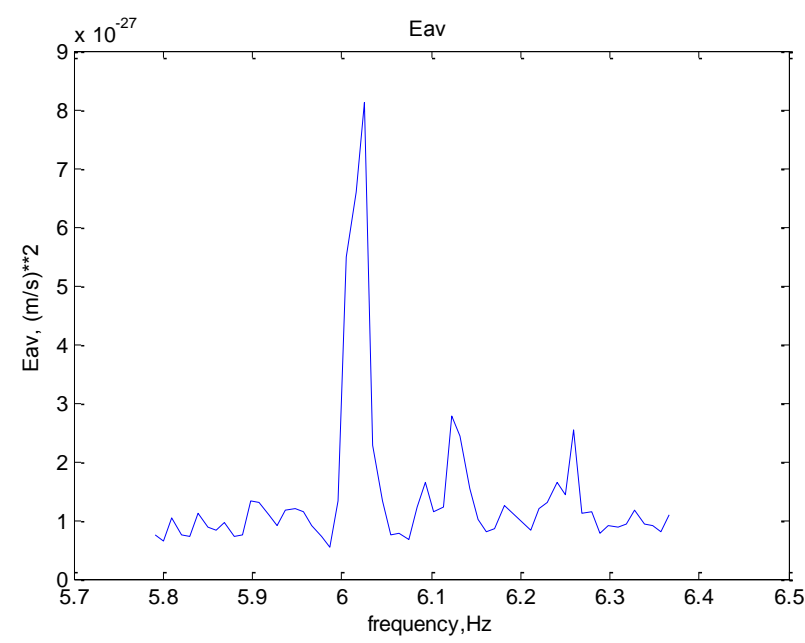

Fig. 3. The average energies EPSP in time and space for 90 hours.

Using the values of $E_{\text {av }}(f)$ (Fig. 3) at the frequencies of the left and right of the band $[5.99 \div 6.05 \mathrm{~Hz}]$, we can estimate the mathematical value of $M\left(E_{\mathrm{av}}\right) \approx 1.2 \cdot 10^{-27}(\mathrm{~m} / \mathrm{s})^{2}$, rms $\sigma_{\text {Eav }}$ satisfies: $0.2(\mathrm{~m} / \mathrm{s})^{2} \leq \sigma_{\text {Eav }} \cdot 10^{27} \leq 0.49(\mathrm{~m} / \mathrm{s})^{2}$.

The amplitude of the ejection of $A\left(f_{0}\right) \approx 8 \cdot 10^{-27}(\mathrm{~m} / \mathrm{s})^{2}$ and $\mathbf{S N R}=\boldsymbol{\Delta}\left(\boldsymbol{f}_{\mathbf{0}}\right) / \boldsymbol{\sigma}_{\mathbf{E a v}} \geq 12.8$, where $\Delta\left(f_{0}\right)=A\left(f_{0}\right)-M\left(E_{\mathrm{av}}\right)$.

The error of the 1-st kind :

$$
\alpha \leq P(\xi>12.8)
$$

where $\xi=N(0,1)-$ normally distributed random variable. The value $\boldsymbol{\alpha}$ is close to zero, the confidence level detection signal $\boldsymbol{P}_{\text {conf }}=\mathbf{1}-\boldsymbol{\alpha}$ is close to 1 .

\section{Threshold GSA}

The minimum values $E_{a v}(f)($ fig. 3$)$ determine threshold of GSA at frequencies close to 6 $\mathrm{Hz}:$

- $\boldsymbol{D}_{\mathbf{E P S P}} \approx 10^{-27}(\mathrm{~m} / \mathrm{s})^{2}$ with respect to energy;

- VEPSP $\approx 0.3 \cdot 10^{-13} \mathrm{~m} / \mathrm{s}$ with respect to speed;

- $\mathbf{a}$ EPSP $\approx 0.8 \cdot 10^{-15} \mathrm{~m}$ with respect to displacement.

The amplitude of the velocity and displacement are related by: $\boldsymbol{A}_{\text {displ }}=\boldsymbol{A}_{v} /(2 \pi \cdot f)$. 


\section{The average amplitude of detected signals}

The average energy of the detected signal at a frequency $\mathrm{f}=6.023 \mathrm{~Hz}$ for 90 hours is $\approx 0.8 \cdot 10^{-26}(\mathrm{~m} / \mathrm{s})^{2}$ (Figure 3 ), corresponding to the velocity $\approx 0.9 \cdot 10^{-13} \mathrm{~m} / \mathrm{s}$ and the corresponding displacement:

$$
\boldsymbol{A}_{\text {signal detect }} \approx 0.9 \cdot 10^{-13} \mathrm{~m} / \mathrm{s} /(2 \pi \cdot f)=2.5 \cdot 10^{-15} \mathrm{~m} \text {. }
$$

Thus, it is experimentally confirmed (7) that using the optimal data of global seismic antenna detected relative motion $\left(\approx 10^{-15} \mathrm{~m}\right)$ different points of the Earth.

\section{Sources of periodic gravitational radiation}

The sources of these harmonic signals naturally associated with pulsars. Figure 2 shows a plot of the signal of the radio emission of pulsar PSR $1919+21$. It follows from the stability of the pulsar period, but this property does not apply to the form of these signals. The spectrum of temporary fragment (Figure 4) is shown in Figure 5.

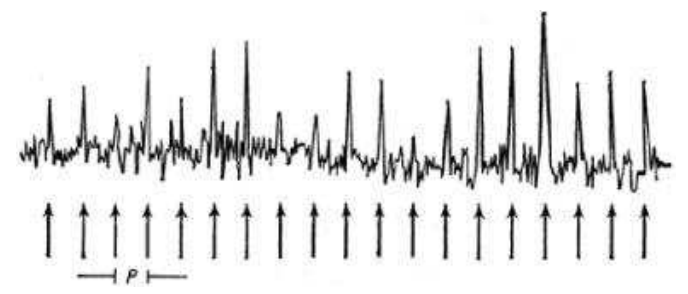

Fig.4. Signals of radio emission PSR $1919+21$ at a frequency of $72.7 \mathrm{MHz}$ [18].

Pulsar period ( $P$ was equal to $1.33730113 \mathrm{~s}$ ) at the time of its opening.

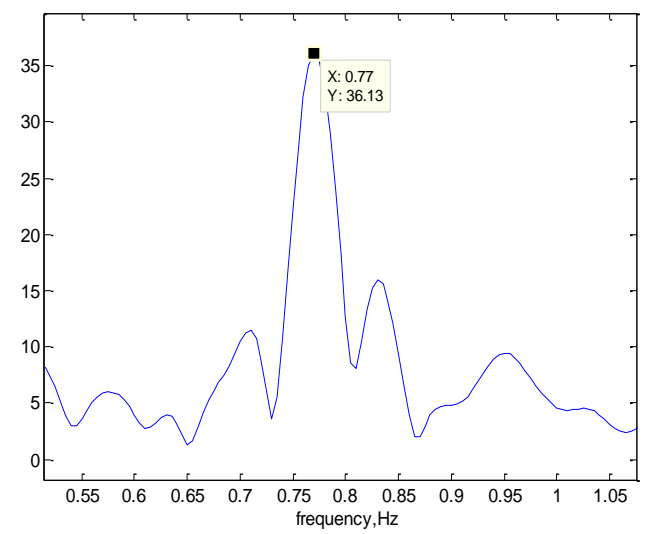

Fig.5. The spectrum of radio emission signals PSR $1919+21$. 
The apparent frequency (Figure 4 ) of these signals $f_{\text {visual }}=1 / \mathrm{T}=1 / 1.33730113=0.747 \mathrm{~Hz}$.

The center frequency $f_{\text {cent }}=0.77 \mathrm{~Hz}$ significantly differs from the apparent frequency, shift $\Delta f \approx 0.023 \mathrm{~Hz}$ that is $\approx 3 \%$ of the center frequency: $\frac{\left|f_{\text {visul }}-f_{\text {cent }}\right|}{f_{\text {cent }}} \approx 3 \%$.

Instability waveforms radio emission is observed for many pulsars $[18,19]$ so we can assume that the radio emission of pulsars observed arbitrary property: center frequency can differ by a few percent of the visible frequency specified in the catalogs of pulsars.

Gravitational wave radiation (GWR) of the pulsar is determined by the quadrupole moment of the source. Given a priori ignorance of the quadrupole moment, we can assume that the complexity of gravitational radiation is similar to the complexity of pulsar radio emission. Consequently, the emission spectrum of GWR is the convolution of the spectra of the modulating function and some of the original signal and this spectrum of GWR expands and "floats" near the center frequency. Most of the signal energy is concentrated in band $\Delta f_{\text {pulsar }} \approx 0.03 \mathrm{~Hz}$.

\section{Characteristics of the detected signals}

Local deformation caused by signal type (2) can be estimated:

$$
h_{0}=D_{l o c}=\left|\frac{\partial \mathbf{S}(\mathbf{r}, t)}{\partial r}\right| \approx \frac{\omega}{c} \sqrt{a_{d i s p}(f, \mathbf{p})^{2}+b_{d i s p}(f, \mathbf{p})^{2}}, \omega=2 \pi f .
$$

Using (3) and (4) can be obtained on frequency signal $f_{0}$ :

$$
h_{0} \approx \frac{\sqrt{E\left(f_{0}, \mathbf{p}\right)}}{c}
$$

The relative change in the distance between stations is

$$
\Delta L_{i, j} \approx \max \left|s\left(\mathbf{r}_{i}, t\right)-s\left(\mathbf{r}_{j}, t\right)\right|, i, j-\text { station numbers. }
$$

If it is unknown the phase shift between the stations, in the worst case $\Delta L_{i, j}$ is less than double the amplitude of the recorded signal.

Deformation (curvature) of space can be estimated by the change in the distance between any stations on the network, for example between stations ZALV and TXAR 


$$
D_{g l}=\frac{\Delta L}{L_{Z A L V-T X A R}} \leq \frac{2 \cdot 2.5 \cdot 10^{-15}}{9498 \cdot 10^{3}} \approx 5.26 \cdot 10^{-22},
$$

where $\boldsymbol{L}_{\text {ZALV-TXAR }}=9498 \mathrm{~km}$.

Given the expression (5), local deformation (strain) for $f=6.023 \mathrm{~Hz}$ can be estimated:

$$
\begin{aligned}
& D_{\text {loc }}=\left|\frac{\partial S(r, t)}{\partial r}\right| \approx \frac{\omega}{c} \sqrt{a(f, \mathrm{p})^{2}+b(f, \mathrm{p})^{2}}=\frac{2.5 \cdot 10^{-15} \cdot 2 \pi \cdot 6.023}{3 \cdot 10^{8}} \approx \\
& \approx 3.15 \cdot 10^{-22} .
\end{aligned}
$$

From a comparison of the two estimates deformation $D_{g l}, D_{l o c}$ it is follows that the velocity of gravitational waves and the speed of light are of equal order.

The estimation of the propagation speed of the GW-wave can be obtained using the relation:

$$
\begin{gathered}
V_{\text {grav }} \approx S_{V} / D_{g l} \\
V_{\text {grav }} \geq 0.9 \cdot 10^{-13} / 5.26 \cdot 10^{-22} \approx 1.7 \cdot 10^{8} \mathrm{~m} / s .
\end{gathered}
$$

In the following will be given direct methods estimate the speed of gravitational waves with better accuracy.

The pulsar J0945-4833 is the most probable source of detected GW-signals, on gravitational radiation expended about $\varepsilon \approx 10^{-5}$ on the total energy of the pulsar.

\section{Conclusion}

With optimal processing of global seismic antenna, relative motions $\left(\approx 10^{-15} \mathrm{~m}\right)$ different points of the Earth were detected, corresponding signals lie in planes perpendicular to the direction of the radiation source, have a high degree of elliptical polarization.

Detection of signals for a long time (90 hours) confirms the fact of registration of the GW-wave in the frequency band near of $6.023 \mathrm{~Hz}$. 
Confidence probability of detection of gravitational-wave is close to 1 .

Characteristics of detected signals (amplitude, center frequency) vary in time, i.e.

GW-signal is quasi-harmonic signal with a smoothly varying parameters. The source of these GW-signals (GW-wave) is periodic (generally quasi-periodic), which is typical for pulsars.

Verified that they cause deformation (curvature) of the order of $10^{-21}$.

The propagation speed of the GW-wave $V_{\text {grav }}>1.7 * 10^{8} \mathrm{~m} / \mathrm{s}$.

The pulsar J0945-4833 is the most probable source of detected GW-signals, on gravitational radiation expended about $\varepsilon \approx 10^{-5}$ on the total energy of the pulsar.

\section{References}

1. Torn K. (2001), Chernye dyry i gravitacionnye volny [Black holes and gravitational waves]. Vestnik RAN [Herald of the RSA], Vol. 71, № 7, 587-590.

2. Misner C.W., Thorne K.S., Wheeler J.A. (1973). Gravitation. San Francisco: W.H. Freeman and Company.

3. Taylor J. (1994). Binary pulsars and relativistic gravity. UFN, Vol. 164, №1, 757-764.

4. Press U., Torn K. (1973). Gravitational wave astronomy. UFN, Vol. 110, №1, 569-603.

5. Grishchuk L.P., Lipunov V.M., Postnov K.A., Prokhorov M.E., Sathyaprakash B.S. (2001). Gravitational wave astronomy: in anticipation of first sources. UFN, Vol. 171, №1, 3-59.

6. The LIGO scientific collaboration. (2009). LIGO: the Laser Interferometer Gravitational-Wave Observatory. Rep. Prog. Phys., Vol.72, №7.

7. Acernese F., Amico P., Arnaud N. (2003). Status of VIRGO. Class. Quantum Grav., Vol.20, S609-S616.

8. Veber J. (1961). General relativity and gravitational waves. Moscow: Publishing house «Foreign literature».

9. Lukanenkov A. V. (2014). Detector gravitational signals. Engineering Physics, №5, 3-15.

10. Lukanenkov A. V. (2014). Experimental and theoretical evaluation of detection of gravitational waves. Engineering Physics, №12, 42-51.

11. Retrieved from: http://www.ctbto.org/fileadmin/content/treaty/treatytext.tt.html.

12. Jaranovski P., Krolak A. (2009). Analysis of gravitational -wave data. Cambridge: Cambridge University Press. 
13. Kedrov O.K. (2005). Seismicheskie metody kontrolya yadernykh ispytanii [Seismic Methods of Monitoring Nuclear Tests]. Moscow, Saransk: IFZ RAN [IPE RAS].

14. Aki K., Richards P.G. (1973). Quantative Seismology. Theory and Methods. Vol.1,2. San Francisco: W.H. Freeman and Company.

15. Fix J. (1972). Ambient earth motion in the period range from 0.01-2560s. BSSA, Vol.62, 1753-1760.

17. Franiti G.E. (1962). The Spectrum of seismic noise. BSSA, Vol.52, 113-121.

18. Hewish A. (1969). Pulsars. UFN, Vol. 97. № 4, 715-732.

19. Manchester R., Taylor J. (1980). Pulsars. Moscow: Publishing house «MIR» [«World»].

Appendix A

\section{Earth as a gravitational wave detector}

A gravitational wave causes a deformation of the bodies, the deformation is very typical of the type. The simplest type - this periodic compression and expansion of the body in two opposite directions.

The intensity of gravitational waves is relative to the distortion of the body, so the more the body itself, the greater will be the absolute value of the deformation. You can measure the local variations of the earth's surface and at the disposal of researchers for a long time, there are devices that detect such fluctuations - seismometers.

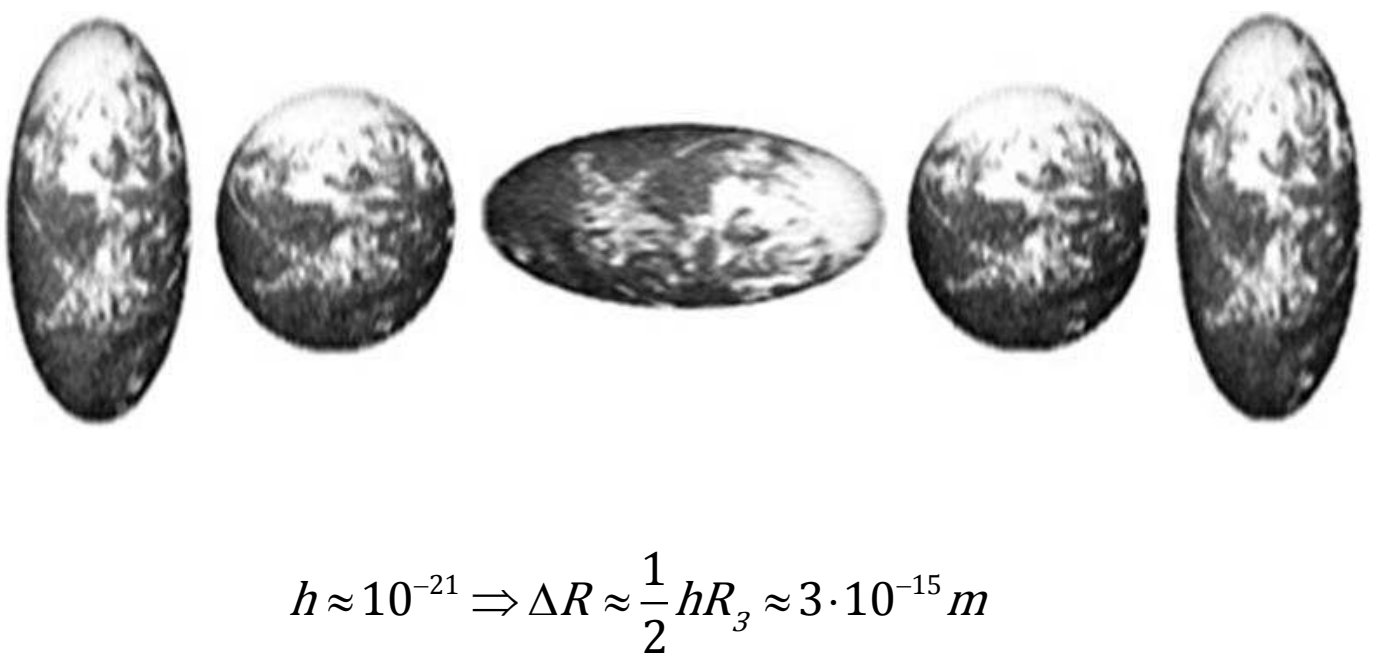

Fig.A1. The deformation of the Earth during the passage of a gravitational wave. 
Earth must be deformed into an ellipsoid in the field of gravitational radiation, extended perpendicular to the direction of the incoming wave.

Vibrations of the earth's surface at $h \approx 10^{-21}$ will be several femtometrov $\left(10^{-15} \mathrm{M}\right)$.

Appendix B

\section{The Association of pulsars}

The frequency of gravitational waves twice the angular speed, $\omega_{p u l}=2 \cdot \omega_{r}$.

The energy of a full rotation of the pulsar:

$$
E_{p u l}=\frac{J \cdot \omega_{p u l}^{2}}{8}, \omega_{r}=2 \pi f_{r}, \omega_{p u l}=2 \pi f_{p u l}, f_{p u l}=2 f_{r},
$$

where $J$ - moment of inertia about the axis of rotation;

$$
\begin{aligned}
& f_{r} \text { - rotation frequency of pulsar ; } \\
& f_{p u l} \text { - frequency of GW-radiation. }
\end{aligned}
$$

Maximum energy emitted per unit frequency interval, in the form of gravitational radiation [1]:

$$
\frac{d E}{d \omega}=\frac{J \omega_{p u l}}{4}
$$

The spectral energy density of the gravitational-wave radiation (upper bound) at a distance $r_{p u l}[1]:$

$$
\Phi_{\text {max }}\left(\omega_{p u l}, r_{p u l}\right)=\frac{\Delta E}{\Delta \omega \cdot 4 \pi \cdot r_{p u l}^{2}}=\frac{J \omega_{p u l}}{4 \cdot 4 \pi \cdot r_{p u l}^{2}}
$$

For a signal from the pulsar registered must be respected [1]:

$$
\Phi_{G W}\left(\omega_{p u l}\right)<\Phi_{\max }\left(\omega_{p u l}, r_{p u l}\right)
$$

This is a necessary condition for registration GW-radiation from the real source is a simple test for the accuracy of detection. 
The amount of energy transferred by GV-waves per unit frequency interval and per unit area (the analogue of the equation (37.31) [2]):

$$
\Phi_{G W}(\omega)=\frac{\aleph \omega^{2}}{8 \pi}\left(\left|\tilde{A}_{+}(\omega)\right|^{2}+\left|\tilde{A}_{\times}(\omega)\right|^{2}\right), \quad \aleph=\frac{c^{3}}{G}
$$

If the source is periodic (for example, if $A_{+}(t)=h_{+}(t)=h_{0} \sin \left(\omega_{0} t\right), h_{\mathrm{x}}=0, \omega_{0}$ - frequency of gravitational radiation), the spectral energy density of the gravitational-wave radiation this source takes the form:

$$
\Phi\left(\omega_{0}\right)=\frac{\aleph \omega_{0}^{2} h_{0}^{2}}{32 \pi}
$$

Using (5), the spectral energy density of gravitational-wave radiation can be represented as:

$$
\Phi\left(\omega_{0}\right)=\Phi_{G W}\left(\omega_{0}\right)=\frac{c \omega_{0}^{2} E\left(f_{0}, \mathbf{p}\right)}{32 \pi G}, \omega_{0}=2 \pi f_{0}
$$

The energy value of the detected signal at the frequency $f=6.023 \mathrm{~Hz}$ :

$$
\Phi_{G W}\left(\omega_{0}\right)=5^{*} 10^{-4} \mathrm{erg} /\left(\mathrm{cm}^{2} \cdot \mathrm{Hz}\right)
$$

Association of pulsars carried by radiation frequency and the position of the registered source on the celestial sphere.

With the source of gravitational-wave radiation near the frequency $f_{0}=6.023 \mathrm{~Hz}$ can be association three pulsars (Table B1) [3,4].

Table B1

\begin{tabular}{|c|c|c|c|}
\hline Pulsar & $\mathrm{L}_{\mathrm{pc}}, \mathrm{kpc}$ & $f_{r}, \mathrm{~Hz}$ & $\Phi_{\max }\left(\omega_{\mathrm{pul}}, \mathrm{r}_{\mathrm{ul}}\right), \mathrm{erg} /\left(\mathrm{cm}^{2} \cdot \mathrm{Hz}\right)$. \\
\hline $\mathrm{B} 1800-27$ & 3.6 & 2.99 & 36.4 \\
\hline $\mathrm{J} 1046+0304$ & 2.25 & 3.06 & 95.3 \\
\hline $\mathrm{J} 0945-4833$ & 2.71 & 3.016 & 64.7 \\
\hline
\end{tabular}


For these pulsars $\varepsilon=\Phi_{G W}\left(\omega_{0}\right) / \Phi_{\max }\left(\omega_{p u l}, r_{p u l}\right) \approx 10^{-5}$, that is expended on gravitational radiation about $\varepsilon \approx 10^{-5}$ on the total energy of the pulsar .

The most probable source is the pulsar J0945-4833.

\section{References B}

1. Zeldovich B.Ya., Novikov I.D. (1971). Theory of gravity and evolution of stars. Moscow: Publishing house «Science».

2. Misner C.W., Thorne K.S., Wheeler J.A. (1973). Gravitation. San Francisco: W.H. Freeman and Company.

3. ATNF Pulsar Catalogue. Retrieved from: http://www.atnf.csiro.au/research/pulsar/psrcat/.

4. Manchester R.N., Hobbs, G.B., Teoh, A., Hobbs, M. (2005). AJ, 129, 1993-2006. 